\title{
El comportamiento del hueco de ventana en la arquitectura vernácula mediterránea española ante las necesidades de acondicionamiento solar
}

\section{Behavior of pit window on to Spanish Mediterranean vernacular conditioning solar needs}

E. Larrumbide $^{(*)}$, C. Bedoya ${ }^{(* *)}$

\section{RESUMEN}

El hueco de ventana es uno de los elementos de acondicionamiento pasivo más importante de la envolvente térmica de una construcción, y por tanto el más sensible al intercambio térmico con el exterior y a las variaciones en las condiciones de soleamiento que se manifiesten en el entorno. La arquitectura vernácula, que cuenta con una amplia representación de tipos en la Península Ibérica, se convierte en el marco adecuado del análisis de la interrelación existente entre la edificación y el entorno, y en concreto entre el hueco de ventana y el soleamiento del lugar. El desarrollo de una metodología que facilite el estudio del hueco de ventana, desde el punto de vista del soleamiento, permite obtener respuestas sobre la mayor o menor adaptación que ofrece el mismo a las necesidades de acondicionamiento solar que se requieran en cada zona climática.

Palabras clave: Factor de sombra; ventana; arquitectura vernácula; retranqueo de ventana.

\section{ABSTRACT}

The window opening is one of the most important elements of the thermal envelope of a building passive conditioning, and therefore more sensitive to heat exchange with the outside and variations in sunlight conditions that manifest in the environment. Vernacular architecture, with a broad representation of types in the Iberian Peninsula, becomes the appropriate framework of analysis of the interrelationship between the building and the environment, and in particular between the window opening and the irradiation of the place. Developing a methodology to facilitate the study of the window opening from the point of view of sunlight, can get answers on the degree of adaptation featuring the same solar conditioning needs that are required in each climate zone.

Keywords: Factor shade; window; vernacular architecture; setback window.

${ }^{(*)}$ Instituto de Ciencias de la Construcción Eduardo Torroja (IETcc-CSIC). Madrid (España).

${ }^{(* *)}$ Escuela Técnica Superior de Arquitectura de Madrid - Universidad Politécnica Madrid (España).

Persona de contacto/Corresponding author: elarrumbide@ietcc.csic.es (E. Larrumbide).

Cómo citar este artículo/Citation: Larrumbide, E., Bedoya, C. (2015). El comportamiento del hueco de ventana en la arquitectura vernácula mediterránea española ante las necesidades de acondicionamiento solar. Informes de la Construcción, 67(539): e105, doi: http://dx.doi.org/10.3989/ic.14.056.

Licencia / License: Salvo indicación contraria, todos los contenidos de la edición electrónica de Informes de la Construcción se distribuyen bajo una licencia de uso y distribución Creative Commons Reconocimiento no Comercial 3.o. España (cc-by-nc). 


\section{OBJETIVOS}

Desde tiempos inmemoriales la arquitectura vernácula ha ofrecido respuesta al clima de formas muy diversas. La arquitectura vernácula española ofrece un amplio catálogo de soluciones edificatorias a lo largo de todo el territorio nacional, convirtiéndose en un amplio campo de observación científica.

Uno de los elementos más representativos de la arquitectura vernácula, que permite caracterizar el comportamiento bioclimático de muchas de las tipologías constructivas de una zona determinada es el hueco de ventana.

Del análisis de su geometría, tamaño y su disposición en la envolvente térmica con respecto al sur geográfico, es posible deducir de forma intuitiva la mayor o menor adecuación que presenta el hueco de ventana, y por tanto su arquitectura, a las necesidades de acondicionamiento solar que se requieren en cada lugar en función de los condicionantes climáticos.

La perduración en el tiempo de una especie o forma, según las teorías evolucionistas, determina una adaptación al entorno; de esta manera, realizando una analogía, aquellas construcciones que permanecen en el tiempo y sobreviven a los rigores climatológicos se encuentran más adaptadas al entorno y proporcionan un mayor grado de cobijo a sus moradores. Modificaciones del entorno climatológico pueden ocasionar alteraciones formales del conjunto edificatorio.

El objetivo que se plantea en este artículo responde a la intención de establecer, a partir de las observaciones realizadas en la arquitectura vernácula mediterránea española, un análisis de la adecuación que presenta el hueco de ventana a las necesidades de acondicionamiento solar en función de las condiciones climáticas de cada lugar.

Para ello se ha desarrollado una metodología de estudio de la ventana basada en establecer una relación entre factor de sombra de la ventana en función del retranqueo y la severidad climática.

Aunque el estudio se ha concretado en una parte de la arquitectura vernácula mediterránea, la metodología empleada puede hacerse extensiva al análisis del hueco de ventana en cualquier edificio y orientación. En el estudio realizado no se han tenido en cuenta otros aspectos relevantes de las ventanas como son la ventilación o la iluminación.

\section{ANTECEDENTES}

Son muchos los autores que han estudiado la importancia del hueco de ventana y su mayor o menor integración en la arquitectura vernácula. Unos han destacado en sus trabajos las características formales que se encuentran en una determinada localidad, como pueden ser aquellos aspectos relacionados con los tamaños de las ventanas, la mejor o peor disposición de huecos en orientaciones dominantes, la utilización del color como elemento de acondicionamiento, etc. Otros sin embargo, plantean el estudio de la arquitectura vernácula desde criterios numéricos, relacionados por ejemplo con la mayor o menor captación de radiación solar a través del hueco acristalado y su incidencia en la demanda energética.

La arquitectura vernácula, como fuente de conocimiento que es, ha sido objeto de múltiples estudios tipológicos en los que se recoge amplia información sobre los materiales empleados en su configuración, las distintas distribuciones en planta que se manifiestan en las diversas zonas geográficas, las particularidades formales de su volumetría e incluso las características constructivas y estructurales que las definen y justifican una mayor o menor adaptación al lugar.

Entre los autores que realizaron extensos estudios sobre la arquitectura vernácula española destacan Luis Feduchi (1) o Carlos Flores (2), en cuyas publicaciones se plasma la riqueza y diversidad que ofrece la arquitectura vernácula española. También se pueden encontrar recientes publicaciones de investigadores sobre la arquitectura vernácula, como las realizadas por Javier de Cárdenas y Chávarri, Luís Maldonado Ramos y Javier Gil Crespo (3), donde se recoge, entre otros aspectos, un interesante muestrario de geometrías de huecos de ventana de la arquitectura vernácula de la isla de Lanzarote. Javier Gil Crespo (4) destaca en su artículo sobre las características de las ventanas canarias, la adecuada respuesta que ofrecen al acondicionamiento bioclimático.

Otros autores también realizan referencias concretas a la forma, tamaño o geometría de los huecos de ventana. Tal es el caso de Ramón Ripoll i Masferrer (5), el cual a través de sus estudios sobre la masía, establece una primera distinción entre los tamaños de las ventanas, considerando que presentan mayor tamaño las ventanas de las masías de zonas húmedas frente a las masías de las zonas cálidas.

Otros autores que han estudiado la arquitectura ibicenca, como Catalina Verdera y Cis Lenaerts (6), destacan entre otros aspectos el tamaño de hueco tan pequeño que se encuentra en la arquitectura vernácula ibicenca.

La profesora Margarita de Luxán García de Diego, con amplia experiencia en el análisis de la arquitectura vernácula española, ha realizado estudios acerca de construcciones vernáculas específicas, en los que se demuestra la importancia que tiene en el acondicionamiento de las viviendas, el hecho de que la vivienda se encuentre orientada hacia el mediodía solar. En el libro Habitar sostenible. Integración medioambiental de 15 casas de arquitectura popular española (7), se analizan múltiples ejemplos de diferentes tipologías vernáculas, observándose cómo, en climas peninsulares con cierto rigor climático en invierno, como es el caso de las Alpujarras, se encuentran huecos de ventana de gran tamaño que se disponen en la orientación sur. Por el contrario se aprecia cómo en climas con un verano más extremo, como el que se registra en la costa almeriense del cabo de Gata, en las viviendas con terrado se dispone un tipo de hueco de ventana de menores dimensiones.

Guillermo Yáñez (8), considera que «Las arquitecturas vernáculas son como sabemos, arquitecturas con un alto grado de adaptación al clima y se podrían llamar, por tanto, arquitecturas fuertemente climáticas. Ello se aprecia fundamentalmente en el tipo de cubierta, superficies de huecos, espesor de muros, materiales, etc..., donde incluso los aspectos solares están integrados».

A través de estos trabajos se pueden constatar las extraordinarias diferencias que se manifiestan en las construcciones vernáculas españolas, así como la mayor o menor capacidad de integración que presenta el hueco de ventana en su arquitectura a lo largo de la amplia y diversa geografía de la Península Ibérica, las islas Canarias o Baleares. 
La importancia de la ventana como elemento de captación solar ha sido destacada por muchos autores, entre los que se indica al profesor Santiago Vega Amado, en su artículo «La ventana colectora» (9).

Otros autores como F. Mustieles (10) reivindicaron la importancia que tiene el hueco de ventana en el acondicionamiento del espacio interior edificado, indicando que debe estar adecuadamente resuelto para lograr efectos beneficiosos en el espacio habitado.

La incidencia del soleamiento en el edificio se considera algo clave hoy en día para lograr un proyecto adaptado a los condicionantes del lugar. En esta línea de estudios se pueden citar los trabajos de Margarita de Luxán y Gloria Gómez en el proyecto de dos bloques de viviendas y locales comerciales en San Cristóbal de los Ángeles en Madrid (11).

Desde un punto de vista técnico, Ylenia Cascone, junto a otros autores (12), establecen un procedimiento de cálculo informático para corregir los errores que plantean muchas normas a la hora de determinar el factor de sombra de las ventanas.

Por este motivo se ha desarrollado una metodología de cálculo que permita obtener el factor de sombra y realizar cálculos más precisos de la radiación solar incidente sobre un hueco acristalado, puesto que las nuevas exigencias normativas, como es el caso del DB HE de 2013 (13) hacen que la ventana sea un elemento importante de acondicionamiento y se requieran cálculos más precisos. Para el desarrollo de la herramienta informática se han empleado las formulaciones sobre geometría solar determinadas por el profesor F. Javier Neila y otros autores (14).

H.D. Cheung y T.M. Chung (15), plantean una metodología alternativa para estudiar la sombra sobre una ventana en un edificio en Hong Kong, mediante la división de la fracción de la semi-bóveda celeste en fracciones.

La importancia del sombreamiento sobre el hueco de ventana y su incidencia en el acondicionamiento interior de la vivienda, ha sido analizado por Ralegaonkar, Rahul V., et al. (16), mediante el análisis del sombreamiento de una ventana empleando un dispositivo estático de control solar en Pilani, Rajasthan (India).

M. K. Urbikain y J. M. Sala (17) desarrollaron una metodología simple para comparar la energía que se obtiene a través de distintas ventanas, determinando las posibilidades de ahorro para distintas zonas climáticas.

En el artículo «Solar radiation calculation methodology for building exterior surfaces» Francisco José Sánchez de la Flor y otros autores (18), exponen que la contribución solar a través de los huecos de ventana es un elemento de acondicionamiento importante y su control en el periodo de verano supone una adecuada estrategia de acondicionamiento en dicho período.

Por lo que respecta a la normativa española, el DB HE de 2013 (13) a través del Documento de Apoyo al Documento Básico DB-HE/1, relativo al cálculo de parámetros característicos de la envolvente (19), incluye tablas en las que se indica el factor de sombra de ventanas para diferentes retranqueos, dimensiones y orientaciones.
En la publicación Guía de diseño integral de viviendas energéticamente eficientes en Andalucía (20), se determinan correlaciones para obtener el factor de sombra medio diario para diferentes latitudes, orientaciones y geometrías de ventana.

Estudios e investigaciones sobre el hueco de ventana y su contribución a la demanda energética de acondicionamiento en función del entorno, se pueden encontrar, por ejemplo, en la tesis doctoral de Francisco José Sánchez de la Flor (21), donde se establece mediante una correlación matemática, la contribución de la radiación solar de la ventana a la demanda energética del edificio, en función de los grados día, la latitud, la declinación solar y el factor solar del vidrio.

En esta tesis mencionada se indica que, para un coeficiente de sombra de valor 1, la influencia de la radiación solar en verano es mayor en el hueco de ventana que en el muro. La contribución de la ventana a la demanda en verano es más alta cuanto mayor sea la severidad climática de dicho periodo. Mientras que en invierno, se manifiesta el mismo fenómeno pero a la inversa, siendo mayor la contribución de la ventana a la disminución de la demanda energética cuanto más alto sea el valor de la severidad climática de invierno.

A raíz de los trabajos expuestos anteriormente se plantea en este artículo, mediante el desarrollo de una nueva metodología, el grado de adaptación que presentan las geometrías de ventana a las necesidades de soleamiento del lugar en relación con sus características climáticas.

\section{METODOLOGÍA DE ESTUDIO}

\subsection{Justificación del estudio}

El hueco de ventana es un sistema de acondicionamiento muy sensible a las condiciones ambientales exteriores, siendo un elemento importante dentro del conjunto edificatorio, en cuanto a la capacidad que ofrece para ventilar, iluminar o transmitir energía.

En el caso del hueco de ventana como elemento captador de radiación solar, éste posee la capacidad de recibir radiación solar y contribuir con ello a disminuir las cargas de calefacción en invierno, o por el contrario, puede incrementar las cargas de refrigeración durante el periodo de verano.

El desarrollo de una metodología numérica de análisis del hueco de ventana en función de la sombra y de las características climáticas del lugar, podría contribuir a facilitar el dimensionado de la ventana para favorecer la disminución de la demanda energética de calefacción y refrigeración, establecida normativamente por el actual DB HE de 2013 (13).

El marco adecuado para plantear el estudio del hueco de ventana es considerar como fuente de datos la arquitectura vernácula de la franja mediterránea española, donde la fuerte impronta del mar Mediterráneo, las variaciones de latitud y altitud, así como las características geográficas específicas de cada localidad, ofrecen un conjunto de subdivisiones climáticas distintas, que inciden de forma directa en los rasgos arquitectónicos que presenta esta arquitectura.

El estudio se centra en las variaciones formales y geométricas entre ventanas de geometría cuadrada y de geometría de 
hueco vertical, para diferentes tamaños de huecos de ventana, limitando el estudio a la orientación sur, con un mismo retranqueo.

Seleccionada la muestra de ventanas en cada zona geográfica, se desarrolla una metodología en la que se plantea el análisis de la sombra que produce el hueco de ventana, o, lo que es lo mismo, el factor de sombra, que se define en el DB HE de 2013 (13), como la fracción de radiación solar incidente en un hueco que no queda bloqueada por la presencia de obstáculos de fachada, tales como voladizos, retranqueos, toldos, salientes laterales u otros. Sus valores oscilan entre 1 y o.

\subsection{Zona geográfica}

Por acotar el área de estudio, la investigación se plantea concretamente en una zona geográfica comprendida entre los paralelos $36^{\circ}$ y $42^{\circ} \mathrm{N}$, aproximadamente, en la zona mediterránea española que cuenta con suficientes variaciones climáticas que condicionan su arquitectura.

Esta zona de estudio pertenece a la zona climática I.3, según la clasificación climática de la Península Ibérica establecida por el meteorólogo Inocencio Font Tullot, la cual se subdivide a su vez en tres sub-zonas, denominadas la zona Noroeste, la Levantina y la Sureste.

Como referencia a lo determinado en el actual DB HE de 2013 (13), se representan gráficamente las zonas climáticas para las capitales de provincia y los climas de referencia, en las que se indican las zonas concretas sobre las que se realizan los estudios, Figura 1, correspondiéndose con la zona A4, B3,
D2 y D1, abarcándose todos los escenarios de veranos posibles (1-4).

También se recoge la representación gráfica de las severidades climáticas de los municipios de la Península Ibérica y las ciudades autónomas de Ceuta y Melilla, en las que se referencian las zonas de estudio contempladas, Figura 2.

\subsection{Desarrollo de una metodología}

Para el estudio del hueco de ventana en función de la sombra en distintas zonas geográficas, ha sido necesario determinar una metodología de análisis numérico que permitiese por un lado, comparar huecos de ventana de tamaños y de geometrías diferenciadas; y por otro lado, que la discriminación de ventanas se realizase en función de las distintas características climáticas de cada zona.

La mejor ventana sería aquella que obtuviese la máxima captación solar para el periodo de invierno y la mínima incidencia solar en el periodo sobrecalentado, en el caso de darse éste.

Para el estudio se considera que el periodo frío o de invierno es el tiempo comprendido entre los meses de octubre a mayo, ambos incluidos; y que el periodo cálido o de verano es el comprendido entre los meses de junio a septiembre, ambos también incluidos.

Para ello se define la expresión [3]:

$$
\left(1-\mathrm{FS}_{\mathrm{I}}\right)+\left(\mathrm{FS}_{\mathrm{V}}\right)
$$

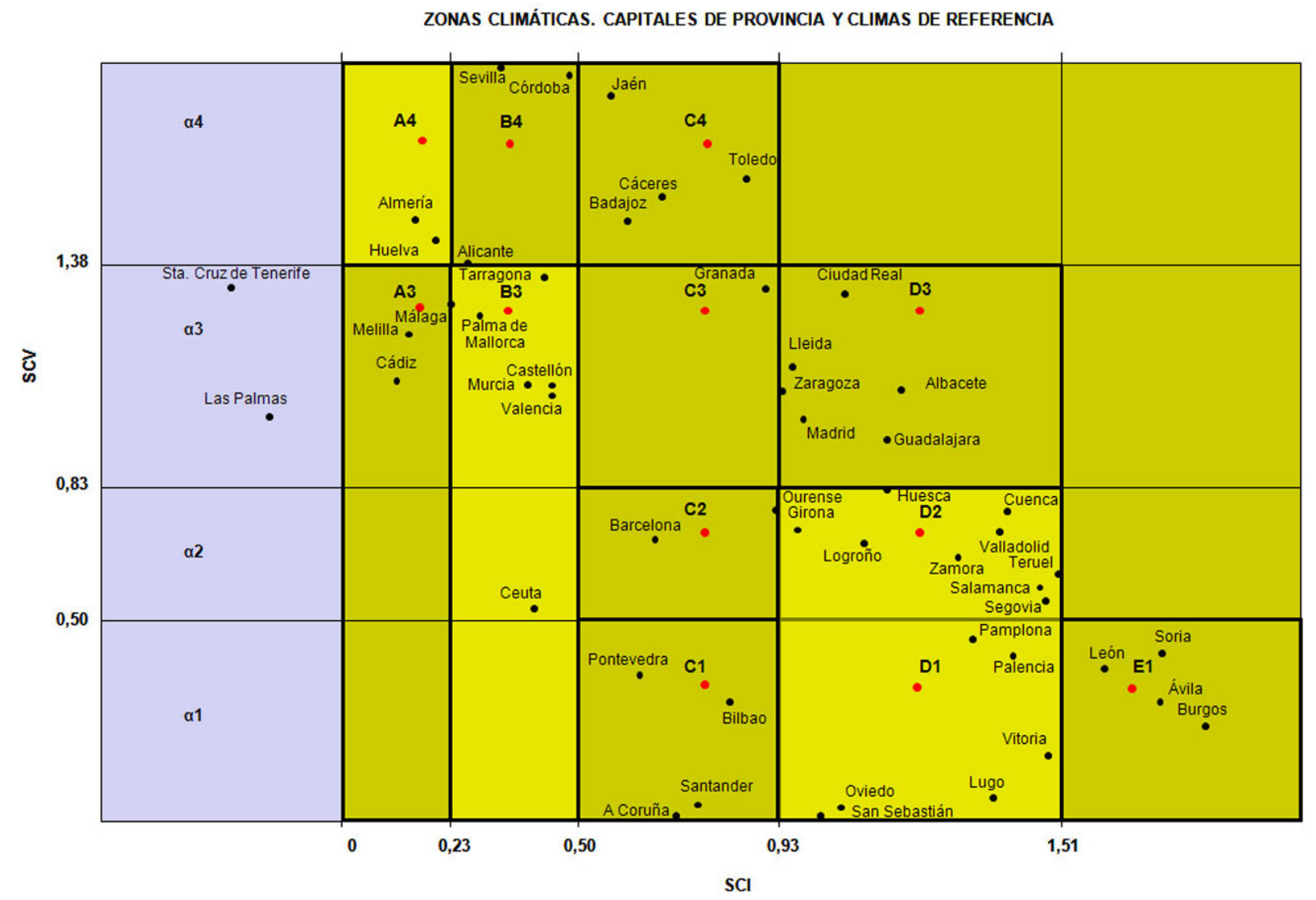

Figura 1. Representación de las zonas climáticas para los climas de referencia y capitales de referencia. 


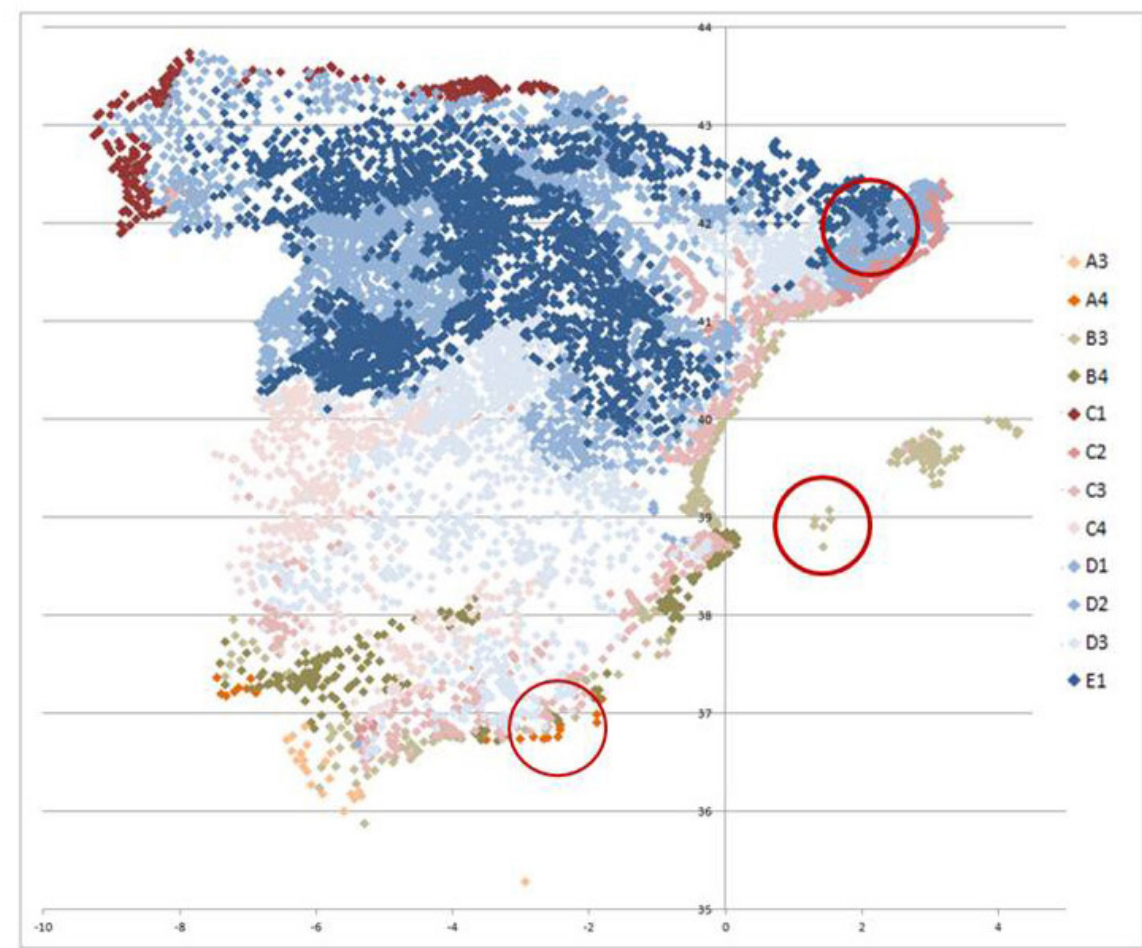

Figura 2. Representación de las severidades climáticas de los municipios de la Península Ibérica, islas Baleares y las ciudades autónomas de Ceuta y Melilla.

Donde,

$\mathrm{FS}_{\mathrm{I}}$, es el factor de sombra medio de los meses de octubre a mayo

$\mathrm{FS}_{\mathrm{V}}$, es el factor de sombra medio de los meses de junio a septiembre

La mejor ventana sería aquella que arrojase el valor más bajo como resultado de la expresión anterior.

Para poder definir un indicador que permita establecer una discriminación de ventanas en función de las necesidades de aprovechamiento solar según las zonas geográficas, se establece una relación ponderada entre la expresión definida anteriormente y la severidad climática de verano e invierno, de acuerdo con la expresión [4]:

$$
\mathrm{IvP}=\mathrm{SCI} \cdot\left(1-\mathrm{FS}_{\mathrm{I}}\right)+\mathrm{SCV} \cdot\left(\mathrm{FS}_{\mathrm{V}}\right)
$$

Donde,

SCI, es la severidad climática del periodo de invierno, considerando el valor medio de los meses de octubre a mayo, ambos incluidos.

SCV, es la severidad climática del periodo de verano, considerando el valor medio de los meses de junio a septiembre, ambos incluidos.

Una vez obtenidos los valores mediante las expresiones anteriores, se delimita un rango u horquilla de resultados entre los menores valores obtenidos con las expresiones [3] $\mathrm{y}[4]$.

Para seleccionar una ventana, los valores obtenidos con las expresiones [3] y [4] se multiplican, tal y como se indica en la fórmula [5], de manera que la mejor ventana sería aquella que menor resultado arroje.

$\mathrm{SVP}=\left[\left(1-\mathrm{FS}_{\mathrm{I}}\right)+\left(\mathrm{FS}_{\mathrm{V}}\right)\right] \cdot\left[\mathrm{SCI} \cdot\left(1-\mathrm{FS}_{\mathrm{I}}\right)+\mathrm{SCV} \cdot\left(\mathrm{FS}_{\mathrm{V}}\right)\right]$

\subsection{Desarrollo de la herramienta de cálculo del factor de sombra}

Para el análisis numérico de la sombra del hueco de ventana se ha procedido al desarrollo de una herramienta informática en soporte Excel, que permitiese la determinación del factor de sombra del hueco de forma horaria o mensual, en cualquier latitud, orientación y en función del retranqueo y de la propia geometría del hueco de ventana.

Esta herramienta desarrollada por el autor de la tesis Optimización energética de la ventana en función de la sombra: el hueco en tipologías de la arquitectura vernácula mediterránea (22), permite, además de la obtención del factor de sombra de la ventana, el dibujo de la sombra de la ventana de forma horaria junto con la obtención de la radiación solar directa, difusa y acumulada. También se obtiene el dibujo de las cartas solares cilíndrica y estereográfica, así como la determinación de las obstrucciones solares de objetos remotos al edificio.

Los resultados obtenidos con la metodología desarrollada, han sido contrastados con los valores del factor de sombra en función del retranqueo determinados en el documento DA DB HE/1, relativo al cálculo de los parámetros característicos (19), junto con los resultados que se pueden obtener mediante la aplicación de las correlaciones matemáticas que se recogen en el documento Guía de diseño integral de viviendas energéticamente eficientes en Andalucía (20). 
Los valores determinados con la nueva herramienta son algo más precisos que los que se obtiene con dichos documentos mencionados.

\subsection{Selección de tipologías arquitectónicas}

Como prototipo de tipología arquitectónica, se ha considerado un tipo de cada una de las zonas climáticas determinadas según la clasificación de Inocencio Font en la franja mediterránea. Así para la zona noroeste se recurre a la masía catalana, en este caso propia de una zona premontañosa; para la zona levantina se elige como tipología edificatoria el casament de la isla de Ibiza; y, finalmente, para la zona sudeste se considera el terrado almeriense.

Para cada tipología de construcción vernácula se selecciona un conjunto de ventanas, para las cuales se obtendrán los factores de sombra medios mensuales mediante la herramienta informática y se aplicará la metodología de selección determinada.

\subsection{Determinación de zonas climáticas}

Cada una de las edificaciones pertenece a una zona climática según el DB HE de 2013 (13). Para la determinación de las clasificaciones climáticas se emplea la Tabla B1 del citado Documento, en la que, en función de la altitud en la que se emplace el edificio, se determina la severidad climática.

Así para las construcciones vernáculas de la zona noreste, situadas a una altitud comprendida entre $450 \mathrm{~m}$ y $600 \mathrm{~m}$, debido a la dispersión geográfica que ofrecen las masías entre Barcelona y Girona y para simplificar el estudio, se consideran dos zonas climáticas la D1 y la D2. Para la zona levantina con una altitud menor de $250 \mathrm{~m}$, la clasificación climática obtenida es la B3. Finalmente para la zona sudeste y una altitud menor de 100 m, la clasificación climática es la A4.

De acuerdo con el DB HE de 2013 (13), se entiende por severidad climática como el cociente entre la demanda energética de un edificio en una localidad y la correspondiente al mismo edificio en una localidad de referencia.
A la hora de realizar el estudio para cada zona climática se emplean los climas de referencia indicados en el Documento descriptivo de climas de referencia (24). Se considera como clima de referencia el clima normalizado que define los parámetros climáticos (temperatura, radiación solar...) representativos de una zona climática concreta para el cálculo de la demanda, permitiéndose con ello estandarizar las solicitaciones exteriores.

Las severidades climáticas de invierno y verano empleadas en las zonas climáticas consideraras son para D1 $(1,2 ; 0,34)$; para D2 $(1,21 ; 0,71)$; para $\mathrm{B} 3(0,35 ; 1,26)$; y para A4 $(0,17 ; 1,69)$.

\subsection{Selección de huecos}

La selección de ventanas se ha agrupado en cinco tipos de huecos de proporción aproximadamente rectangular y otros cinco de proporción cuadrada, lo que se considera una muestra suficientemente representativa para plantear el análisis, pudiendo hacerse extensivo el estudio a cualquier otro conjunto de huecos. Para simplificar el análisis se considera un retranqueo único de $30 \mathrm{~cm}$ para todas las ventanas consideradas.

Las geometrías de los huecos seleccionados en cada zona son las que se definen en la Tabla 1.

Para el conjunto de ventanas seleccionadas se han determinado los factores de sombra medios mensuales y anuales para la orientación sur. Los cálculos se han realizado para el día 15 de cada mes.

Posteriormente, se ha considerado una ventana representativa de cada grupo, eligiendo un valor medio de tamaño de hueco, con lo que se plantea un estudio para seleccionar la ventana que presente mejor comportamiento solar, variando exclusivamente la geometría del hueco entre la forma de ventana cuadrada y la vertical.

De esta manera, para las zonas D1 y D2 se considera una ventana con superficie media de $1,00 \mathrm{~m}^{2}$, para la zona B3 una de $0,35 \mathrm{~m}^{2}$ y para la A4 una de $0,50 \mathrm{~m}^{2}$.

Tabla 1. Geometrías de huecos seleccionados. Zonas D1, D2, B3 y A4.

\begin{tabular}{|l|c|c|c|c|c|c|c|c|c|c|}
\hline D1/D2 & a1 & a2 & a3 & a4 & a5 & a6 & a7 & a8 & a9 & a10 \\
\hline H (horizontal) & 1,30 & 1,00 & 1,00 & 0,90 & 0,80 & 0,82 & 0,80 & 0,70 & 0,60 & 0,40 \\
\hline V (vertical) & 1,50 & 1,42 & 1,30 & 1,20 & 1,13 & 1,10 & 1,05 & 0,95 & 0,90 & 0,60 \\
\hline Proporción $(\mathrm{V} / \mathrm{H})$ & 1,15 & 1,42 & 1,30 & 1,33 & 1,41 & 1,34 & 1,31 & 1,36 & 1,50 & 1,50 \\
\hline Superficie & 1,95 & 1,42 & 1,30 & 1,08 & 0,90 & 0,90 & 0,84 & 0,67 & 0,54 & 0,24 \\
\hline
\end{tabular}

\begin{tabular}{|l|c|c|c|c|c|c|c|c|c|c|}
\hline B3 & a1 & a2 & a3 & a4 & a5 & a6 & a7 & a8 & a9 & a10 \\
\hline H (horizontal) & 0,60 & 0,50 & 0,55 & 0,40 & 0,35 & 0,90 & 0,60 & 0,55 & 0,50 & 0,45 \\
\hline V (vertical) & 0,84 & 0,80 & 0,77 & 0,64 & 0,49 & 0,80 & 0,60 & 0,55 & 0,50 & 0,45 \\
\hline Proporción $(\mathrm{V} / \mathrm{H})$ & 1,40 & 1,60 & 1,40 & 1,60 & 1,40 & 0,89 & 1,00 & 1,00 & 1,00 & 1,00 \\
\hline Superficie & 0,50 & 0,40 & 0,42 & 0,26 & 0,17 & 0,72 & 0,36 & 0,30 & 0,25 & 0,20 \\
\hline
\end{tabular}

\begin{tabular}{|l|c|c|c|c|c|c|c|c|c|c|}
\hline A4 & a1 & a2 & a3 & a4 & a5 & a6 & a7 & a8 & a9 & a10 \\
\hline H (horizontal) & $\mathrm{o}, 80$ & 0,60 & 0,60 & 0,60 & 0,50 & 0,80 & 0,80 & 0,90 & 0,60 & 0,55 \\
\hline V (vertical) & 1,00 & 0,75 & 0,80 & 0,75 & 0,60 & 0,80 & 0,75 & 0,80 & 0,50 & 0,50 \\
\hline Proporción $(\mathrm{V} / \mathrm{H})$ & 1,25 & 1,25 & 1,33 & 1,25 & 1,20 & 1,00 & 0,94 & 0,89 & 0,83 & 0,91 \\
\hline Superficie & 0,80 & 0,45 & 0,48 & 0,45 & 0,30 & 0,64 & 0,60 & 0,72 & 0,30 & 0,28 \\
\hline
\end{tabular}




\section{RESULTADOS}

En primer lugar, para ambos estudios realizados, los resultados se presentan de forma completa para la zona D1, resumiéndose, en segundo lugar, los valores obtenidos para todas las zonas climáticas consideradas.

En la Tabla 2 se resumen los resultados obtenidos de los factores de sombra medios mensuales y anuales, así como los datos obtenidos con los indicadores propuestos con los que se permite realizar la selección de ventana.

De acuerdo con el indicador determinado que selecciona la ventana con mejor captación solar para ambos periodos, ponderada en función de la severidad climática, la mejor ventana sería la a1, con una geometría de 1,30 × 1,50 $\mathrm{m}^{2}$ (ancho $\times$ alto) y una superficie de $1,95 \mathrm{~m}^{2}$. La horquilla de selección se encuentra entre la a1 y la a4.

En la Tabla 3 se analiza el distinto comportamiento que presenta una ventana con la misma superficie y diferentes proporciones, variándose entre la proporción cuadrada y la vertical.

La mejor ventana, en este caso, sería la ventana a11 con una superficie de $1,00 \mathrm{~m}^{2}$ y una geometría de $0,95 \times 1,05 \mathrm{~m}^{2}$. La horquilla de mejor ventana se sitúa entre la a1o y la a11.

En la Tabla 4 se resumen las características de las ventanas que presentan mejor comportamiento según la metodología descrita en todas las zonas consideradas, para el estudio realizado con diferentes geometrías y tamaños de huecos.

La representación gráfica de los factores de sombra medios se encuentra en la Figura 3.

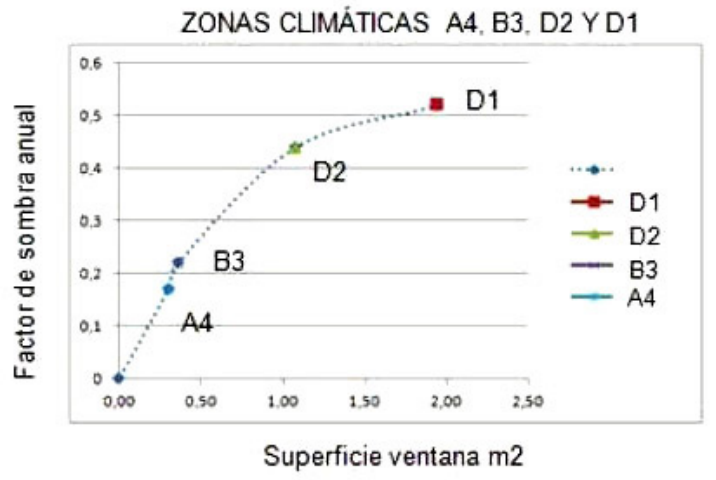

Figura 3. Factor de sombra medio anual para ventanas con diferentes superficies y geometrías.

La ecuación polinómica que se ha obtenido como resultado del estudio realizado para ventanas con diferentes tamaños y geometrías de ventanas, que se ha representado en la Figura 3, es la indicada en la expresión [6]:

$$
\begin{gathered}
\text { Fsm }=-0,19141243+1,54968293 \times \\
\times \mathrm{S}-1,24851564 \times \mathrm{S}^{2}+0,32866521 \times \mathrm{S}^{3}
\end{gathered}
$$

Donde,

Fsm, es el factor de sombra medio anual.

$\mathrm{S}$, es la superficie del hueco.

En la Tabla 5 se recogen las características más significativas de las mejores ventanas del estudio realizado para las ventanas de tamaños medios, en el que se mantiene la superficie del hueco y se modifica la proporción de la ventana, pasando de una geometría cuadrada a otras con proporción vertical.

Tabla 2. Resultados de selección de ventanas con distinta geometría y superficie. Zona D1.

\begin{tabular}{|l|c|c|c|c|c|c|c|c|c|c|}
\cline { 2 - 10 } \multicolumn{1}{c|}{ Zona D1 } & $\mathbf{a 1}$ & $\mathbf{a 2}$ & $\mathbf{a 3}$ & $\mathbf{a 4}$ & $\mathbf{a 5}$ & $\mathbf{a 6}$ & $\mathbf{a 7}$ & $\mathbf{a 8}$ & $\mathbf{a 9}$ & $\mathbf{a 1 0}$ \\
\hline H (horizontal) & 1,30 & 1,00 & 1,00 & 0,90 & 0,80 & 0,82 & 0,80 & 0,70 & 0,60 & 0,40 \\
\hline V (vertical) & 1,50 & 1,42 & 1,30 & 1,20 & 1,13 & 1,10 & 1,05 & 0,95 & 0,90 & 0,60 \\
\hline
\end{tabular}

\begin{tabular}{|l|c|c|c|c|c|c|c|c|c|c|}
\hline Enero & 0,77 & 0,72 & 0,71 & 0,69 & 0,65 & 0,66 & 0,65 & 0,60 & 0,55 & 0,47 \\
\hline Febrero & 0,64 & 0,56 & 0,55 & 0,51 & 0,57 & 0,57 & 0,56 & 0,51 & 0,46 & 0,37 \\
\hline Marzo & 0,59 & 0,52 & 0,51 & 0,47 & 0,42 & 0,43 & 0,41 & 0,46 & 0,41 & 0,31 \\
\hline Abril & 0,39 & 0,44 & 0,42 & 0,37 & 0,34 & 0,33 & 0,31 & 0,26 & 0,30 & 0,09 \\
\hline Mayo & 0,29 & 0,34 & 0,30 & 0,25 & 0,22 & 0,20 & 0,18 & 0,12 & 0,09 & 0,00 \\
\hline Junio & 0,25 & 0,21 & 0,16 & 0,12 & 0,14 & 0,12 & 0,08 & 0,04 & 0,00 & 0,00 \\
\hline Julio & 0,23 & 0,27 & 0,23 & 0,18 & 0,15 & 0,14 & 0,11 & 0,09 & 0,06 & 0,00 \\
\hline Agosto & 0,43 & 0,37 & 0,34 & 0,30 & 0,26 & 0,26 & 0,24 & 0,27 & 0,23 & 0,04 \\
\hline Septiembre & 0,54 & 0,46 & 0,44 & 0,40 & 0,47 & 0,47 & 0,45 & 0,40 & 0,35 & 0,24 \\
\hline Octubre & 0,60 & 0,51 & 0,50 & 0,57 & 0,53 & 0,53 & 0,52 & 0,47 & 0,41 & 0,32 \\
\hline Noviembre & 0,75 & 0,70 & 0,70 & 0,67 & 0,63 & 0,64 & 0,63 & 0,58 & 0,53 & 0,45 \\
\hline Diciembre & 0,79 & 0,74 & 0,73 & 0,70 & 0,68 & 0,68 & 0,67 & 0,63 & 0,58 & 0,39 \\
\hline
\end{tabular}

\begin{tabular}{|l|c|c|c|c|c|c|c|c|c|c|}
\hline Media & 0,52 & 0,49 & 0,47 & 0,44 & 0,42 & 0,42 & 0,40 & 0,37 & 0,33 & 0,22 \\
\hline
\end{tabular}


Tabla 3. Selección de ventanas con la misma superficie y diferente geometría. Zona D1.

\begin{tabular}{|l|c|c|c|c|c|c|c|c|c|c|}
\cline { 2 - 12 } \multicolumn{1}{c|}{ Zona D1 } & $\mathbf{a 1 0}$ & $\mathbf{a 1 1}$ & $\mathbf{a 1 2}$ & $\mathbf{a 1 3}$ & $\mathbf{a 1 4}$ & $\mathbf{a 1 5}$ & $\mathbf{a 1 6}$ & $\mathbf{a 1 7}$ & $\mathbf{a 1 8}$ & $\mathbf{a 1 9}$ \\
\hline H (horizontal) & 1,00 & 0,95 & 0,90 & 0,84 & 0,78 & 0,75 & 0,70 & 0,65 & 0,60 & 0,55 \\
\hline V (vertical) & 1,00 & 1,05 & 1,11 & 1,19 & 1,27 & 1,33 & 1,42 & 1,53 & 1,66 & 1,81 \\
\hline
\end{tabular}

\begin{tabular}{|l|c|c|c|c|c|c|c|c|c|c|}
\hline Enero & 0,69 & 0,68 & 0,68 & 0,67 & 0,66 & 0,65 & 0,64 & 0,62 & 0,59 & 0,57 \\
\hline Febrero & 0,53 & 0,52 & 0,50 & 0,48 & 0,58 & 0,57 & 0,56 & 0,54 & 0,51 & 0,48 \\
\hline Marzo & 0,46 & 0,46 & 0,46 & 0,45 & 0,44 & 0,43 & 0,53 & 0,53 & 0,51 & 0,50 \\
\hline Abril & 0,33 & 0,35 & 0,35 & 0,36 & 0,36 & 0,36 & 0,35 & 0,35 & 0,47 & 0,47 \\
\hline Mayo & 0,17 & 0,19 & 0,22 & 0,24 & 0,27 & 0,28 & 0,29 & 0,29 & 0,30 & 0,30 \\
\hline Junio & 0,05 & 0,09 & 0,13 & 0,11 & 0,14 & 0,15 & 0,17 & 0,19 & 0,32 & 0,34 \\
\hline Julio & 0,09 & 0,12 & 0,14 & 0,17 & 0,20 & 0,21 & 0,23 & 0,24 & 0,25 & 0,25 \\
\hline Agosto & 0,26 & 0,26 & 0,28 & 0,28 & 0,29 & 0,29 & 0,41 & 0,41 & 0,41 & 0,41 \\
\hline Septiembre & 0,39 & 0,39 & 0,39 & 0,49 & 0,49 & 0,48 & 0,48 & 0,47 & 0,46 & 0,44 \\
\hline Octubre & 0,47 & 0,57 & 0,56 & 0,55 & 0,54 & 0,53 & 0,51 & 0,50 & 0,47 & 0,57 \\
\hline Noviembre & 0,67 & 0,67 & 0,66 & 0,65 & 0,64 & 0,63 & 0,62 & 0,60 & 0,58 & 0,55 \\
\hline Diciembre & 0,71 & 0,71 & 0,70 & 0,69 & 0,68 & 0,67 & 0,66 & 0,64 & 0,62 & 0,59 \\
\hline
\end{tabular}

\begin{tabular}{|l|c|c|c|c|c|c|c|c|c|c|c|c|}
\hline Media & 0,40 & 0,42 & 0,42 & 0,43 & 0,44 & 0,44 & 0,45 & 0,45 & 0,46 & 0,46 \\
\hline FSv & 0,20 & 0,22 & 0,23 & 0,26 & 0,28 & 0,28 & 0,32 & 0,33 & 0,36 & 0,36 \\
\hline FSi & 0,50 & 0,52 & 0,52 & 0,51 & 0,52 & 0,52 & 0,52 & 0,51 & 0,51 & 0,51 \\
\hline (1-FSi)+FSv & $\mathbf{0 , 6 9}$ & 0,69 & 0,71 & 0,75 & 0,75 & 0,76 & 0,80 & 0,81 & 0,85 & 0,85 \\
\hline IvP & 0,66 & $\mathbf{0 , 6 5}$ & 0,66 & 0,67 & 0,67 & 0,67 & 0,68 & 0,70 & 0,71 & 0,71 \\
\hline SVP & 0,45 & $\mathbf{0 , 4 5}$ & 0,47 & 0,50 & 0,50 & 0,52 & 0,54 & 0,57 & 0,60 & 0,61 \\
\hline
\end{tabular}

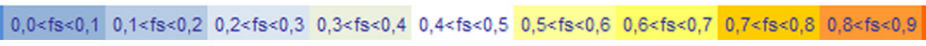

Tabla 4. Resumen de características técnicas de las ventanas con mejor comportamiento en cada zona climática. Estudio para ventanas con distinta superficie y diferente geometría.

\begin{tabular}{|c|c|c|c|c|c|}
\hline Zona climática & Ancho & Alto & Superficie & Proporción & Fs medio año \\
\hline D1 & 1,30 & 1,50 & 1,95 & 1,15 & 0,52 \\
\hline D2 & 0,90 & 1,20 & 1,08 & 1,33 & 0,44 \\
\hline B3 & 0,60 & 0,60 & 0,36 & 1,00 & 0,22 \\
\hline A4 & 0,60 & 0,50 & 0,30 & 0,83 & 0,17 \\
\hline
\end{tabular}

Tabla 5. Resumen de características técnicas de las ventanas con mejor comportamiento en cada zona climática. Estudio para ventanas con misma superficie y distinta proporción.

\begin{tabular}{|c|c|c|c|c|c|}
\hline Zona climática & Ancho & Alto & Superficie & Proporción & Fs medio año \\
\hline D1 & 0,95 & 1,05 & 1,00 & 1,10 & 0,42 \\
\hline D2 & 1,00 & 1,00 & 1,00 & 1,00 & 0,40 \\
\hline B3 & 0,56 & 0,63 & 0,35 & 1,13 & 0,22 \\
\hline A4 & 0,71 & 0,71 & 0,50 & 1,00 & 0,25 \\
\hline
\end{tabular}

Para las zonas climáticas más extremas que se registran en el área analizada (zona D1 y zona A4) se representan gráficamente en la Figura 4 los resultados del segundo estudio. En dicha figura se comparan los valores de los factores de sombra medio anual con el factor de sombra del periodo frío Fsi y del periodo de verano Fsv, para un hueco de ventana con una misma superficie pero diferentes proporciones.

Se aprecia en las figuras una tendencia diferente en el comportamiento de la ventana entre el periodo de verano y el de invierno, teniendo mayor pendiente la línea que representa el factor de sombra del periodo de verano, frente a la tendencia más uniforme que presenta la ventana en el periodo de invierno.

\section{CONCLUSIONES}

Desde un punto de vista general, de acuerdo con la metodología empleada en la que se analiza exclusivamente el comportamiento del hueco de ventana desde el punto de vista del soleamiento, se aprecia en primer lugar que, de acuerdo con las diferentes geometrías y tamaños de ventana estudiados para la orientación sur, a medida que se disminuye de latitud el tamaño de hueco de ventana es más reducido, pasando de la proporción vertical a la proporción aproximadamente cuadrada.

La disminución del tamaño de ventana con la latitud se podría entender, al margen de otras consideraciones constructivas, hi- 

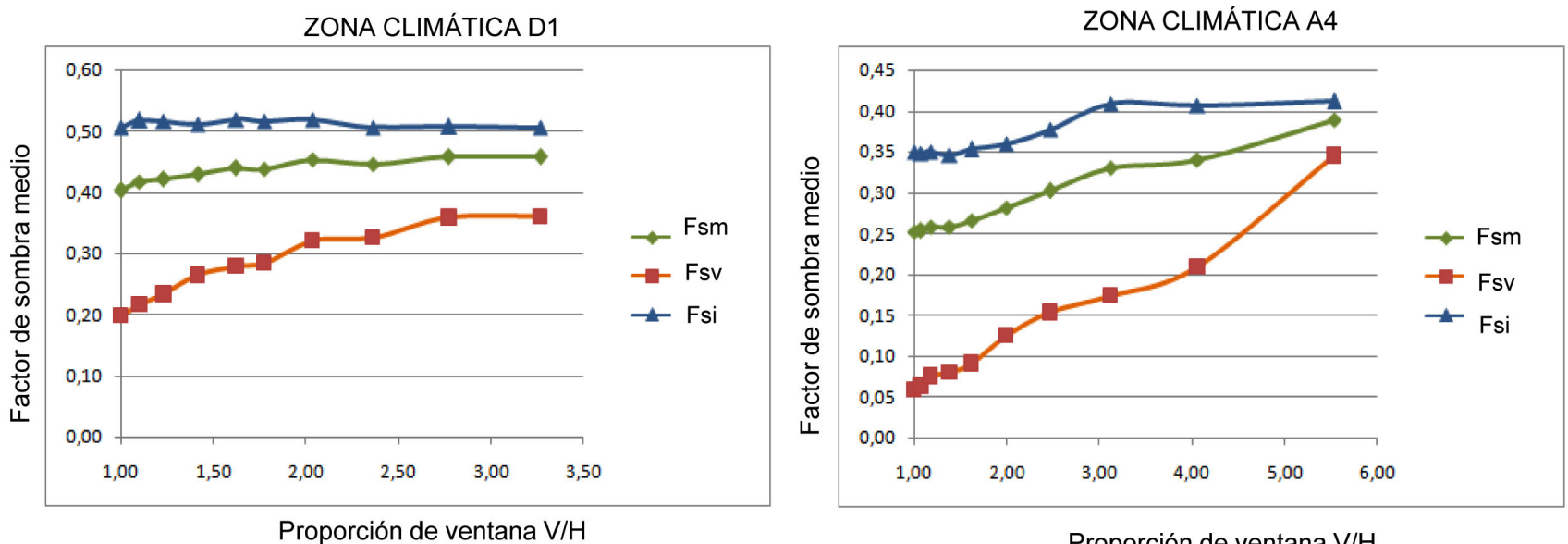

Proporción de ventana V/H

Figura 4. Factor de sombra medio anual para ventanas con misma superficie y diferentes geometrías.

giénicas o lumínicas, por la suavización de la severidad climática de invierno y el incremento de la severidad climática de verano que se produce en zonas de menor latitud, por lo que la arquitectura como estrategia de acondicionamiento pasivo, ofrece un tamaño de ventana de menores dimensiones para evitar el exceso de soleamiento que se registra en el periodo cálido.

En zonas con mayor severidad climática de invierno (D1), la arquitectura responde con un tamaño de hueco de mayores dimensiones, donde el invierno es más riguroso y el verano es más suave, tal y como se ha indicado en la Figura 3, favoreciéndose con ello la captación solar.

A nivel local, estos resultados obtenidos guardan cierta relación con lo recogido por Ramón Ripoll i Masferrer (5), que diferenciaba entre las ventanas de mayor tamaño que se daban en las masías de las zonas húmedas, frente al tamaño de hueco más reducido que se apreciaba en las masías de las zonas más soleadas.

En la zona climática B3, desde el punto de vista del soleamiento, se observa un tamaño de ventana excesivamente pequeño con respecto a las dimensiones de los huecos de ventana del resto de zonas consideradas. Esta dimensión reducida del hueco de ventana, se puede justificar entre otros motivos por la necesidad disponer un hueco que satisfaga las necesidades de ventilación que se requieren en esta zona, tal y como recogen diversos autores en el libro Arquitectura y espacio rural en Ibiza (6), no adaptándose por contra dicho tamaño de hueco a las necesidades de soleamiento que se requieren para el periodo frío. En este sentido Catalina Verdera, historiadora de Arte, y Cis Lenaerts, arquitecto justifican, en el citado libro anterior (6), el tamaño reducido de los huecos por el ambiente cálido que se registra durante el verano, siendo preferible el ambiente en penumbra. Esta autora indica en sus estudios que la forma de la ventana es generalmente de proporciones cuadradas, hecho que se manifiesta en el estudio realizado.
Esta particularidad de los huecos de ventana en la arquitectura ibicenca, en la que se aprecia un mal comportamiento de la ventana a las necesidades de soleamiento en el periodo frío, donde se registra un exceso de sombra, podría justificar el carácter importado de esta arquitectura propia de climas de latitudes inferiores o de Asia menor, como indican, entre otros, Raoul Haussmann, Eric Muhle o Blastak en el libro también citado anteriormente (6).

Por lo que respecta al estudio realizado en el que se varía la proporción de hueco de ventana entre la geometría vertical y la cuadrada con igualdad de superficie, la metodología empleada permite extraer como conclusión para la orientación sur, que la ventana de geometría cuadrada es la más adecuada desde el punto de vista de las necesidades de soleamiento anuales en todas las zonas climáticas de la franja de estudio considerada, excepto en aquellas zonas de elevada altitud con severidad climática alta en invierno y baja en verano (D1), donde resulta algo más interesante la ventana de geometría vertical, ya que se necesita un cierto nivel de recepción solar en el periodo de verano. La diferencia fundamental de comportamiento entre una ventana de geometría vertical y otra de geometría cuadrada, a igualdad de superficie y retranqueo, para el conjunto de ventanas referenciadas, es que con la primera, proporción vertical, se registra mayor captación solar en el periodo cálido, de junio a septiembre, siendo muy parecida o ligeramente mayor la captación solar que se obtiene con ésta en el periodo frío, de octubre a mayo, frente a una ventana de proporciones cuadradas, que ofrece mayor sombra en el periodo estival, Figura 4. Esta metodología desarrollada permite establecer una discriminación de ventanas de forma sencilla sin plantear complejos cálculos de balance térmico.

Parte de los datos recogidos en este artículo se pueden ver en la tesis Optimización energética de la ventana en función de la sombra: el hueco en tipologías de la arquitectura vernácula mediterránea (22). 


\section{REFERENCIAS}

(1) Feduchi, L. M. (1978). Itinerarios de arquitectura popular española. Barcelona: Ed. Blume-Labor.

(2) Flores López, C. (1986). Arquitectura popular española (5 vols). Madrid: Ed. Aguilar.

(3) Cárdenas y Chávarri, J. de, Maldonado-Ramos, L., Gil-Crespo, I.J. (2007). Arquitectura Popular de Lanzarote. Madrid: Fundación Diego de Sagredo.

(4) Gil Crespo, I. J. (2014). El lenguaje vernáculo de las ventanas tradicionales canarias: antecedentes. Tipología y funcionamiento bioclimático. Anuario de Estudios Atlánticos. (60): 817-858.

(5) Ripoll i Masferrer, R. (1983). Les masies de les comarques de Girona. Girona: Ed. Gòthia.

(6) Benjamín, W., et al. (2002). Arquitectura y espacio rural en Ibiza (Cuarta edición ampliada). Eivissa: Demarcació d’Eivissa i Formentera del Col-legi d'Arquitectes de les Illes Balears.

(7) Luxán García de Diego, M.; et al. (2011). Habitar sostenible. Integración medioambiental de 15 casas de arquitectura popular española. Madrid: Centro de Publicaciones Secretaría General Técnica Ministerio de Fomento.

(8) Yáñez Parareda, G. (1982). Energía solar edificación y clima. Tomos I y II. Madrid: Ministerio de Obras Públicas y Urbanismo.

(9) Vega-Amado, S. (1990). La ventana colectora. Informes de la Construcción, 42(409): 75-82, doi: http://dx.doi. org/10.3989/ic.1990.v42.i409.1445.

(10) Mustieles, F., La Roche, P., Machado, M. V., Oteiza, I., Indriago, J. A., González, R. (1998). Cerramientos bioclimáticos para climas húmedos: La cuarta vivienda. Informes de la Construcción, 49(453): 11-22, doi: http://dx.doi.org/10.3989/ ic.1998.v49.i453.917.

(11) Luxán García de Diego, M., Gómez, G. (2006). Dos bloques de viviendas y locales comerciales en San Cristóbal de los Ángeles, Madrid. Informes de la Construcción, 58(502): 5-16, doi: http://dx.doi.org/10.3989/ic.2006.v58.i502.383.

(12) Cascone, Y., Corrado, V., Serra, V. (2011). Calculation procedure of shading factor under complex boundary conditions. Solar Energy, 85(10): 2524-2539, doi: http://dx.doi.org/10.1016/j.solener.2011.07.011.

(13) Ministerio de Fomento. (2013, 12 de septiembre). Orden FOM/1635/2013, de 10 de septiembre, por la que se actualiza el Documento Básico DB-HE «Ahorro de Energía», del Código Técnico de la Edificación, aprobado por Real Decreto 314/2006, de 17 de marzo. Boletín Oficial del Estado, no 219, pp. 67137-67209. España.

(14) Neila-González, F. J. (2013). Acondicionamiento ambiental y habitabilidad del espacio arquitectónico. Madrid: Munilla-Lería.

(15) Cheung, H.D., Chung, T.M. (2007). Analyzing sunlight duration and optimum shading using a sky map. Building and Environment, 42(9): 3138-3148, doi: http://dx.doi.org/10.1016/j.buildenv.2006.07.030.

(16) Ralegaonkar, R. V., Gupta, R. (2005). Design development of static sunshade small scale modelling technique. Renewable Energy, 30(6): 867-880, doi: http://dx.doi.org/10.1016/j.renene.2004.08.004.

(17) Urbikain, M. K., Sala, J. M. (2009). Analysis of different models to estimate energy savings related to windows in residential buildings. Energy and Buildings, 41(6): 687-695, doi: http://dx.doi.org/10.1016/j.enbuild.2009.01.007.

(18) Sánchez-de la Flor, F. J., Ortíz-Cebolla, R., Molina, J. L., Álvarez-Domínguez, S. (2005). Solar radiation calculation methodology for building exterior surfaces. Solar Energy, 79(5): 513-522, doi: http://dx.doi.org/10.1016/j.solener.2004.12.007.

(19) Ministerio de Fomento. (2013). Cálculo de parámetros característicos de la envolvente. En Documento de Apoyo al Documento Básico DB-HE Ahorro de Energía (DA DB-HE/1). Madrid: Ministerio de Fomento.

(20) Grupo de Termotecnia de la Asociación de Investigación y Cooperación Industrial de Andalucía (AICIA). (2012). Guía de diseño integral de viviendas energéticamente eficientes en Andalucía. Sevilla: Ed. Agencia Andaluza de la Energía. Consejería de Economía, Innovación y Ciencia.

(21) Sánchez-de la Flor, F. J. (2002). Modificaciones microclimáticas inducidas por el entorno del edificio y su influencia sobre la demanda energética de acondicionamiento (Tesis). Sevilla: Departamento de Ingeniería Energética y Mecánica de Fluidos. Escuela Superior de Ingenieros - Universidad de Sevilla.

(22) Larrumbide Gómez-Rubiera, E. (2010). Optimización energética de la ventana en función de la sombra: el hueco en tipologías de la arquitectura vernácula mediterránea (Tesis doctoral). Madrid: Universidad Politécnica de Madrid Escuela Técnica Superior de Arquitectura de Madrid. UPM-ETSAM.

(23) Ministerio de Vivienda. (2006, 28 de marzo). Real Decreto 314/2006 de 17 de marzo. Código Técnico de la Edificación. Boletín Oficial del Estado, no 74, pp. 11816-11831. España.

(24) Ministerio de Fomento. (2013). Documento descriptivo de climas de referencia. Madrid: Ministerio de Fomento. 